\title{
Solving Multi-Objective Linear Programming Problems with Fuzzy Interval Based on Decomposition Method
}

\author{
Mohamed Solomon \\ Ph.D. Researcher, Department of Operations Research, Faculty of Graduate Studies for \\ Statistical Research, Cairo University, Giza, Egypt, E-Mail: eng.mohamed666@yahoo.com \\ Mohamed Saied Abd-Alla
}

M.Sc. Researcher, Department of Operations Research, Faculty of Graduate Studies for Statistical Research, Cairo University, Giza, Egypt.

\begin{abstract}
Most of the problems in the real world situations have a multi-objective, in these situations available information in the system are not exact or imprecise. In this paper, solving multiobjective linear programming problems with fuzzy non-negative interval such as objective function coefficients, technical coefficients and fuzzy variables by using an approximation but convenient method called decomposition method has been proposed. In the composition method, ranking functions are not used. With the help of numerical examples, the method is illustrated.
\end{abstract}

Keywords:multi-objective linear programming, fuzzy interval, fuzzy number, fuzzy linear programming, decomposition method.

\section{Introduction}

Multi-objective problems occur in many engineering, scientific research projects, and economics, like risk, cost, time minimization or quality, efficiency, and revenue maximization. These are difficult but practical problems which normally happen. Linear programming [2] in many fields has applications of operations research. It is concerned with the optimization of a linear function while satisfying a set of linear equality and/or inequality constraints or restrictions. Multi-objective linear programming has the same assumptions as ordinary linear programming; their objectives and constraint set must be of the $=, \leq$, or $\geq$ type, and fractional values for the decision variables are allowed.

In real world situation the available information (data) in the system under consideration are not exact, therefore, fuzzy linear programming (FLP) was introduced and studied by many 
researchers[14, 15, 5, 4, 8, 10, 9, 12] Fuzzy set theory has become an important tool in the branch of decision making sciences and has been applied to many disciplines such as control theory and management sciences, mathematical modeling and industrial applications.

The concept of fuzzy decision was introduced by [3], later [14] first extended the concept as fuzzy linear programming (FLP) problems on a general level. Fuzzy linear programming problems have an essential role in fuzzy modeling, which can formulate uncertainty in the real world. Afterwards, many researchers have considered various types of FLP problems and proposed several approaches for solving these problems. In effect, the most convenient methods are based on the concept of comparison of fuzzy numbers by use of ranking functions [4, 10, 9]. Usually in such methods, researchers define a crisp model which is equivalent to the FLP problem and then use the optimal solution of the model as the optimal solution of the FLP problem. [11]Use linear ranking functions for solving fuzzy variable linear programming that uses simplex tableau used to solve linear programming problem in crisp environment. [9]Introduced a dual simplex algorithm for solving linear programming problem with fuzzy variables and its dual by using a general linear ranking function and linear programming directly.

All the above researchers considered either the technical coefficients or the variables as fuzzy, and both [1]. Most problems in the real life have multi-objective and sometimes the available information (data) in the system are not exact. Where human estimation is used are inexact, so as the decision that are taken based on this information. Thus, it is desirable to consider the model with multi-objective function and fuzzy coefficients such as objective coefficients, technical coefficients (L.H.S), and variables (R.H.S). The proposed method in this paper, namely decomposition method, used by [13], to solve integer linear programming problem with fuzzy variables, for solving multi-objective linear programming problem with fuzzy objective coefficients, fuzzy technical coefficients and fuzzy variables.

\section{Preliminaries}

We need the following definitions and theorems to establish the method which can be found at [1].

\subsection{Fuzzy Numbers}

Definition 1: A fuzzy number $\alpha$ is a convex normalized fuzzy set on the real line R such that:

a. There exists at least one $x_{o} \in R$ with $\mu_{\bar{\alpha}}\left(x_{o}\right)=1$.

b. $\mu_{\bar{\alpha}}(\mathrm{x})$ is piecewise continuous

The membership function of any fuzzy number $\alpha$ is as follows:

$$
\mu_{\alpha}(x)=\left\{\begin{array}{lr}
f_{x}, & x \in a, b \\
1, & x \in b, c \\
g_{x}, & x \in a, b \\
0, & \text { otherwise }
\end{array}\right\}
$$

Where $a \leq b \leq c, f$ is increasing and right-continuous function on $[a, b]$, and $g$ is decreasing and left-continuous function on $[b, c]$. If $b=c$, then $a$ is a fuzzy number otherwise it is known as fuzzy interval. 
Definition 2: A fuzzy number $\bar{a}$ is a Triangular Fuzzy Number (TFN) denoted by $a_{1}, a_{2}, a_{3}$ where $a_{1}, a_{2}, a_{3}$ are real numbers and its membership function $\mu_{\alpha}(x)$ is given below.

$$
\mu_{\alpha}(x)=\left\{\begin{array}{cc}
\frac{x-a_{1}}{a_{2}-a_{1}}, & a_{1} \leq x \leq a_{2} \\
\frac{a_{3}-x}{a_{3}-a_{2}}, & a_{2} \leq x \leq a_{3} \\
0, & \text { otherwise }
\end{array}\right\}
$$

Definition 3:A fuzzy number $a=\left\{x, \mu_{\alpha}(x), \mathrm{x} \in \mathrm{R}\right\}$ is non-negative and denoted by $a \geq 0$ if and only if $\mu_{\alpha}(x)=0$ for all $x<0$. Then a TFN is non-negative $a=\left(a_{1}, a_{2}, a_{3}\right)$ if and only if $a_{1} \geq 0$.

Definition 4:A fuzzy matrix $A=\left[a_{i j}\right] m \times n$ is called non-negative if $a_{i j} \geq 0$, for all $i, j$, where $a_{i j}$ 's are fuzzy numbers.

\subsection{Arithmetic on Fuzzy Numbers}

Definition 5:Let $a$ and $b$ be any fuzzy numbers and let * denote any of the four basic arithmetic operations. Then we define a fuzzy set on $\mathbb{R}, a A * b B$ by defining its $a$ - cuts, $a_{a A * b B}=a_{a A B} * a_{b B}$ for any $a \in(0,1]$. When $*=/$, clearly we have to require that $0 \notin$ $a_{b B}$ for all $a \in(0,1]$.

Since $a_{a A * b B}$ is a closed interval for each $a \in(0,1]$ and $a, b$ are fuzzy numbers, $a A * b B$ is also fuzzy numbers, which followed by the following theorem.

Theorem 2.2:Let $* \in\{+,-, \mathrm{x}, /\}$, and let $a, b$ denote any continuous fuzzy numbers. Then the fuzzy set $a A * b B$ defined by

$$
a A * b B z=\sup _{z=x * y} \min \left[\mu_{\alpha}(x) * \mu_{b}(y)\right]
$$

is a continuous fuzzy number.

Proof. Klir and Yuan [7].

Definition 6:Let $a=\left(a_{1}, a_{2}, a_{3}\right)$ and $b=\left(b_{1}, b_{2}, b_{3}\right)$ be two TFNs.

Then
1) $a+b=\left(a_{1}, a_{2}, a_{3}\right)+\left(b_{1}, b_{2}, b_{3}\right)=\left(a_{1}+b_{1}, a_{2}+b_{2}, a_{3}+b_{3}\right)$
2) $a-b=\left(a_{1}, a_{2}, a_{3}\right)+\left(b_{1}, b_{2}, b_{3}\right)=\left(a_{1}-b_{1}, a_{2}-b_{2}, a_{3}-b_{3}\right)$
3) a) $k a=k\left(a_{1}, a_{2}, a_{3}\right)=\left(k a_{1}, k a_{2}, k a_{3}\right)$ for $k \geq 0$
b) $k a=k\left(a_{1}, a_{2}, a_{3}\right)=\left(k a_{3}, k a_{2}, k a_{1}\right)$ for $k \leq 0$
4) $a \cdot b=\left(a_{1}, a_{2}, a_{3}\right) .\left(b_{1}, b_{2}, b_{3}\right)=\left(a_{1} . b_{1}, a_{2} . b_{2}, a_{3} . b_{3}\right) a, b$ are non-negative TFN's.

Let $F(R)$ be the set of all real TFNs.

Definition 7: Let $A=\left(a_{1}, a_{2}, a_{3}\right)$ and $B=\left(b_{1}, b_{2}, b_{3}\right)$ then

- $A=B \Leftrightarrow a_{i}=b_{i}$ for all $i=1$ to 3

- $A \leq B \Leftrightarrow a_{i} \leq b_{i}$ for all $i=1$ to 3

\section{MOLP with Fuzzy Interval}

Consider the following multi-objective linear programming problem with fuzzy objective coefficients, fuzzy technical coefficients and fuzzy variables 


$$
\begin{aligned}
& \left(\mathrm{p}_{\mathrm{o}}\right) \max \widetilde{z_{1}}=\widetilde{c_{1}} \tilde{x} \\
& \max \widetilde{z_{2}}=\widetilde{c_{2}} \tilde{x^{2}} \\
& \max \widetilde{z_{3}}=\widetilde{c_{3}} \tilde{x}
\end{aligned}
$$

s.t

$$
\widetilde{A X} \leq \tilde{b} \quad \tilde{x} \geq 0
$$

Where the coefficient matrix $\tilde{A}=\left(\widetilde{a_{i j}}\right) m \times n$ is real fuzzy matrix, and the coefficient vector $\tilde{C}=\left(\widetilde{c_{j}}\right) n \times 1$ is a non-negative fuzzy vector, and $\tilde{X}=\left(\widetilde{x_{j}}\right) n \times 1$ and $\tilde{b}=\left(\widetilde{b_{i}}\right) m \times 1$ are a nonnegative real fuzzy vectors such that $\widetilde{X_{J}}, \widetilde{b_{i}} \in f(R)$ for all $1 \leq j \leq n$ and $1 \leq i \leq m$

Let objective $1 Z_{A}=\widetilde{c_{1}} \tilde{x}$, objctive $2 Z_{B}=\widetilde{c_{2}} \tilde{x}$, objctive $3 Z_{C}=\widetilde{c_{3}} \tilde{x}, Z_{T}$ is the new objective function after transformed multi-objective functions into a single objective by used weighted method.Supposed that the weighting coefficientsw $\mathbf{w}_{\mathbf{i}}$ are real numbers such that $\mathbf{w}_{\mathbf{i}} \geq 0$. Then the single objective will become as follows

$$
\begin{gathered}
\text { (P) } Z_{T}=\left[w_{1} Z_{A}+w_{2} Z_{B}+w_{3} Z_{C}\right] X \\
A X \leq b \\
X \geq 0 \\
\text { where } w_{1}+w_{2}+w_{3}=1 \\
w_{1}, w_{2}, w_{3} \geq 0
\end{gathered}
$$

(Theorem 2.2.3. and the proof of weighted method in Kaisa Miettinen [6])

Definition 8: A fuzzy vector $\tilde{x}$ is said to be a feasible solution of the problem (P) if $\tilde{x}$ satisfies (3).

Definition 9: A feasible solution $\tilde{x}$ of the problem (P) is said to be an optimal solution of the problem (P) if there exists no feasible $\tilde{u}=\left(\widetilde{u_{j}}\right) n \times 1$ of $(\mathrm{P})$ such that $c \tilde{u}>c \tilde{x}$.

After used the theorem 1 in[13] and the arithmetic operations of fuzzy numbers, we can obtain the following result.

Theorem3.1: A fuzzy vector $\tilde{x}=\left(x_{1}^{o}, x_{2}^{o}, x_{3}^{o}\right)$ is an optimal solution of the problem (P) if and only if $x_{1}^{o}, x_{2}^{o}$, and $x_{3}^{o}$ are optimal solutions of the following crisp linear programming problems (P2), (P1) and (P3) respectively where

$$
\begin{gathered}
\text { (P2) } Z_{T 2}=\left[w_{1} Z_{A 2}+w_{2} Z_{B 2}+w_{3} Z_{C 2}\right] X_{2} \\
A^{2} X_{2} \leq b_{2} \\
X_{2} \geq 0 \\
\text { where } w_{1}+w_{2}+w_{3}=1 \\
w_{1}, w_{2}, w_{3} \geq 0 \\
\text { (P1) } Z_{T 1}=\left[w_{1} Z_{A 1}+w_{2} Z_{B 1}+w_{3} Z_{C 1}\right] X_{1} \\
A^{1} X_{1} \leq b_{1} \\
X_{1} \leq X_{2} \\
X_{1} \geq 0 \\
w_{1}+w_{2}+w_{3}=1 \\
w_{1}, w_{2}, w_{3} \geq 0 \\
\text { where } \\
\text { (P3) } Z_{T 3}=\left[w_{1} Z_{A 3}+w_{2} Z_{B 3}+w_{3} Z_{C 3}\right] X_{3} \\
A^{3} X_{3} \leq b_{3}
\end{gathered}
$$




$$
\begin{gathered}
X_{3} \geq X_{2} \\
X_{3} \geq 0 \\
\text { where } w_{1}+w_{2}+w_{3}=1 \\
w_{1}, w_{2}, w_{3} \geq 0
\end{gathered}
$$

Where $A^{K}=a_{i j}^{K}, \quad k=1,2,3$

Proof: the problem $\left(\mathrm{p}_{\mathrm{o}}\right)$ can be written in the following form

$$
\left[\left(w_{1} C_{1}^{1}+w_{2} C_{2}^{1}+w_{3} C_{3}^{1}\right) X_{1}, \stackrel{(\mathrm{AP}) \max \left(Z_{T 1}, Z_{T 2}, Z_{T 3}\right)=}{\left.\left(w_{1} C_{1}^{2}+w_{2} C_{2}^{2}+w_{3} C_{3}^{2}\right) X_{2},\left(w_{1} C_{1}^{3}+w_{2} C_{2}^{3}+w_{3} C_{3}^{3}\right) X_{3}\right]}\right.
$$

S.T

$$
\begin{gathered}
\left(A^{1} X^{1}, A^{2} X^{2}, A^{3} X^{3}\right) \leq\left(b^{1}, b^{2}, b^{3}\right) \\
x_{1}, x_{2}, x_{3} \geq 0 \\
A^{1}, A^{2}, A^{3} \geq 0 \\
\text { Where } w_{1}+w_{2}+w_{3}=1 \\
w_{1}, w_{2}, w_{3} \geq 0
\end{gathered}
$$

Let $x^{o}=\left(x_{1}^{o}, x_{2}^{o}, x_{3}^{o}\right)$ be an optimal solution of the problem (AP) and $x=\left(x_{1}, x_{2}, x_{3}\right)$ be a feasible solution of the problem $(\mathrm{P})$. This implies that

$$
\begin{gathered}
\left(w_{1} C_{1}^{1}+w_{2} C_{2}^{1}+w_{3} C_{3}^{1}\right) X_{1} \leq\left(w_{1} C_{1}^{1}+w_{2} C_{2}^{1}+w_{3} C_{3}^{1}\right) X_{1}^{O} \\
\left(w_{1} C_{1}^{2}+w_{2} C_{2}^{2}+w_{3} C_{3}^{2}\right) X_{2} \leq\left(w_{1} C_{1}^{2}+w_{2} C_{2}^{2}+w_{3} C_{3}^{2}\right) X_{2}^{O} \\
\left(w_{1} C_{1}^{3}+w_{2} C_{2}^{3}+w_{3} C_{3}^{3}\right) X_{3} \leq\left(w_{1} C_{1}^{3}+w_{2} C_{2}^{3}+w_{3} C_{3}^{3}\right) X_{3}^{O} \\
\left(A^{1} X^{1} \leq b^{1}, A^{2} X^{2} \leq b^{2}, A^{3} X^{3} \leq b^{3}\right) ; x_{1}^{o}, x_{2}^{o}, x_{3}^{o} \geq 0 \\
\text { Where } w_{1}+w_{2}+w_{3}=1 \\
w_{1}, w_{2}, w_{3} \geq 0
\end{gathered}
$$$$
\max Z_{T 1}=\left(w_{1} C_{1}^{1}+w_{2} C_{2}^{1}+w_{3} C_{3}^{1}\right) X_{1}^{O} ; \max Z_{T 2}=\left(w_{1} C_{1}^{2}+w_{2} C_{2}^{2}+w_{3} C_{3}^{2}\right) X_{2}^{O} ; \max Z_{T 3}=
$$$$
\left(w_{1} C_{1}^{3}+w_{2} C_{2}^{3}+w_{3} C_{3}^{3}\right) X_{3}^{O}
$$

Now, from (6) and (7) we can conclude that $x_{1}^{o}, x_{2}^{o}$ and $x_{3}^{o}$ are optimal solutions of the crisp LP problems (AP2), (AP1) and (AP3).

Suppose that $x_{1}^{o}, x_{2}^{o}$ and $x_{3}^{o}$ are optimal solutions of the crisp LP problems (AP2), (AP1) and (AP3) with optimal values $z_{1}, z_{2}$ and $z_{3}$ respectively. This implies that $x^{o}=\left(x_{1}^{o}, x_{2}^{o}, x_{3}^{o}\right)$ is an optimal solution of the problem (AP) with optimal value $z^{o}=\left(z_{1}^{o}, z_{2}^{o}, z_{3}^{o}\right)$ and since (AP) is approximately equivalent to $(\mathrm{P})$, therefore $x^{o}=\left(x_{1}^{o}, x_{2}^{o}, x_{3}^{o}\right)$ is an approximate optimal solution of the problem (P) and hence the result.

\section{Algorithm and Methodology}

Consider the MOLP problem $\left(\mathrm{p}_{\mathrm{o}}\right)$ with fuzzy objective coefficients, fuzzy technical coefficients and fuzzy variables

Step 1:choose $w_{i}$ where $\sum_{i=1}^{n} w_{i}=1, w_{i} \geq 0$ depends on the number of objective functions, then construct crisp LP problem

$$
\begin{aligned}
& \text { (P2) } Z_{T 2}=\left[w_{1} C_{1}^{2}+w_{2} C_{2}^{2}+w_{3} C_{3}^{2}\right] x^{2} \\
& A^{2} x^{2} \leq b^{2} \\
& x^{2} \geq 0
\end{aligned}
$$


and let $x^{2^{o}}$ be optimal solution of the problem (P2)

Step 2: construct crisp LP problem

$$
\begin{aligned}
& \text { (P1) } Z_{T 1}=\left[w_{1} C_{1}^{1}+w_{2} C_{2}^{1}+w_{3} C_{3}^{1}\right] x^{1} \\
& A^{1} x^{1} \leq b^{1} \\
& x^{1} \leq x^{2} \\
& x^{1} \geq 0
\end{aligned}
$$

and let $x^{1^{o}}$ be optimal solution of the problem (P1)

Step 3: construct crisp LP problem

$$
\begin{aligned}
& \text { (P3) } Z_{T 3}=\left[w_{1} C_{1}^{3}+w_{2} C_{2}^{3}+w_{3} C_{3}^{3}\right] x^{3} \\
& A^{3} x^{3} \leq b^{3} \\
& x^{3} \geq x^{2} \\
& x^{3} \geq 0
\end{aligned}
$$

and let $x^{3^{\circ}}$ be optimal solution of the problem (P3)

Then the optimal solution of the original $\left(\mathrm{p}_{\mathrm{o}}\right)$ problem is $x^{o}=\left(x^{1^{o}}, x^{2^{o}}, x^{3^{o}}\right)$.

\section{Numerical Examples}

To illustrate this method two numerical examples are presented

\subsection{Example 1}

S.t

$$
\begin{gathered}
(p) \max Z_{A}=[5,8,12] x_{1}+[6,9,15] x_{2} \\
\max Z_{B}=[8,13,18] x_{1}+[12,19,25] x_{2} \\
\max Z_{C}=[12,16,22] x_{1}+[18,22,28] x_{2}
\end{gathered}
$$

$$
\begin{gathered}
{[10,13,15] x_{1}+[13,16,20] x_{2} \leq[200,325,480]} \\
{[8,10,13] x_{1}+[28,31,37] x_{2} \leq[350,520,735]} \\
x_{1}, x_{2} \geq 0
\end{gathered}
$$

Let $\widetilde{Z_{T}}=\left(Z_{T 1}, Z_{T 2}, Z_{T 3}\right), \widetilde{x_{1}}=\left(y_{1}, x_{1}, t_{1}\right), \widetilde{x_{2}}=\left(y_{2}, x_{2}, t_{2}\right)$

Now the problem (p2) is given blow with $\left(w_{1}=\frac{1}{3}, w_{2}=\frac{1}{3}, w_{3}=\frac{1}{3}\right)$

$$
\begin{gathered}
(p 2) \max Z_{A 2}=\left(8 x_{1}+9 x_{2}\right) \frac{1}{3} \\
\max Z_{B 2}=\left(13 x_{1}+19 x_{2}\right) \frac{1}{3} \\
\max Z_{C 2}=\left(16 x_{1}+22 x_{2}\right) \frac{1}{3} \\
Z_{T 2}=\left(8 x_{1}+9 x_{2}\right) \frac{1}{3}+\left(13 x_{1}+19 x_{2}\right) \frac{1}{3}+\left(16 x_{1}+22 x_{2}\right) \frac{1}{3} \\
Z_{T 2}=12.33 x_{1}+16.67 x_{2}
\end{gathered}
$$

s.t

$$
\begin{gathered}
13 x_{1}+16 x_{2} \leq 325 \\
10 x_{1}+31 x_{2} \leq 520 \\
x_{1}, x_{2} \geq 0
\end{gathered}
$$

Now, using an algorithm for LP problem, the solution of the problem (p2) is 


$$
x_{1}=7.22, \quad x_{2}=14.44, \quad Z_{T 2}=329.84
$$

Now, the problem (p1) is given below

$$
\begin{gathered}
(p 1) \max Z_{A 1}=\left(5 y_{1}+6 y_{2}\right) \frac{1}{3} \\
\max Z_{B 1}=\left(8 y_{1}+12 y_{2}\right) \frac{1}{3} \\
\max Z_{C 1}=\left(12 y_{1}+18 y_{2}\right) \frac{1}{3} \\
Z_{T 1}=\left(5 y_{1}+6 y_{2}\right) \frac{1}{3}+\left(8 y_{1}+12 y_{2}\right) \frac{1}{3}+\left(12 y_{1}+18 y_{2}\right) \frac{1}{3} \\
Z_{T 1}=8.33 y_{1}+12 y_{2}
\end{gathered}
$$

s.t

$$
\begin{gathered}
10 y_{1}+13 y_{2} \leq 325 \\
8 y_{1}+28 y_{2} \leq 520 \\
y_{1} \leq 7.22 \\
y_{2} \leq 14.44 \\
y_{1}, y_{2} \geq 0
\end{gathered}
$$

Now, using an algorithm for LP problem, the solution of the problem (p1) is

$$
y_{1}=5.97, \quad y_{2}=10.8, \quad Z_{T 1}=179.24
$$

Now, the problem (p3) is given below

s.t

$$
\begin{gathered}
(p 3) \max Z_{A 3}=\left(12 t_{1}+15 t_{2}\right) \frac{1}{3} \\
\max Z_{B 3}=\left(18 t_{1}+25 t_{2}\right) \frac{1}{3} \\
\max Z_{C 3}=\left(22 t_{1}+28 t_{2}\right) \frac{1}{3} \\
Z_{T 3}=\left(12 t_{1}+15 t_{2}\right) \frac{1}{3}+\left(18 t_{1}+25 t_{2}\right) \frac{1}{3}+\left(22 t_{1}+28 t_{2}\right) \frac{1}{3} \\
Z_{T 3}=17.33 t_{1}+22.67 t_{2}
\end{gathered}
$$

$$
\begin{gathered}
15 t_{1}+20 t_{2} \leq 480 \\
13 t_{1}+37 t_{2} \leq 735 \\
t_{1} \geq 7.22 \\
t_{2} \geq 14.44 \\
t_{1}, t_{2} \geq 0
\end{gathered}
$$

Now, using an algorithm for LP problem, the solution of the problem (p3) is

$$
t_{1}=12.75, \quad t_{2}=14.44, \quad Z_{T 3}=548.25
$$

Therefore, the solution for the given MOLP problem with fuzzy objective coefficients, fuzzy technical coefficients and fuzzy variables is

$$
\begin{gathered}
\widetilde{x_{1}}=\left(y_{1}, x_{1}, t_{1}\right)=(5.97,7.22,12.75) \\
\widetilde{x_{2}}=\left(y_{2}, x_{2}, t_{2}\right)=(10.8,14.44,14.44) \\
\widetilde{Z_{T}}=\left(Z_{T 1}, Z_{T 2}, Z_{T 3}\right)=(179.24,329.84,548.25)
\end{gathered}
$$

\subsection{Example 2}

$$
\begin{gathered}
\text { (p) } \max Z_{A}=[3,6,12] x_{1}+[4,8,13] x_{2} \\
\max Z_{B}=[5,8,14] x_{1}+[3,7,12] x_{2}
\end{gathered}
$$


s.t

$$
\min Z_{C}=[2,6,9] x_{1}+[4,7,9] x_{2}
$$

$$
\begin{gathered}
{[5,9,15] x_{1}+[4,7,10] x_{2} \leq[40,117,270]} \\
{[4,7,13] x_{1}+[14,17,21] x_{2} \leq[100,207,420]} \\
x_{1}, x_{2} \geq 0
\end{gathered}
$$

Let $\widetilde{Z_{T}}=\left(Z_{T 1}, Z_{T 2}, Z_{T 3}\right), \widetilde{x_{1}}=\left(y_{1}, x_{1}, t_{1}\right), \widetilde{x_{2}}=\left(y_{2}, x_{2}, t_{2}\right)$

Now the problem $(\mathrm{p} 2)$ is given blow with $\left(w_{1}=\frac{1}{3}, w_{2}=\frac{1}{3}, w_{3}=\frac{1}{3}\right)$

$$
\begin{gathered}
(p 2) \max Z_{A 2}=\left(6 x_{1}+8 x_{2}\right) \frac{1}{3} \\
\max Z_{B 2}=\left(8 x_{1}+7 x_{2}\right) \frac{1}{3} \\
\min Z_{C 2}=\left(6 x_{1}+7 x_{2}\right) \frac{1}{3}
\end{gathered}
$$

(Must transform $Z_{C 2}$ to max) $\max Z_{C 2}=\left(-6 x_{1}-7 x_{2}\right) \frac{1}{3}$

$$
\begin{gathered}
\text { (all max) } Z_{T 2}=\left(6 x_{1}+8 x_{2}\right) \frac{1}{3}+\left(8 x_{1}+7 x_{2}\right) \frac{1}{3}+\left(-6 x_{1}-7 x_{2}\right) \frac{1}{3} \\
Z_{T 2}=2.67 x_{1}+2.67 x_{2}
\end{gathered}
$$

S.t

$$
\begin{gathered}
9 x_{1}+7 x_{2} \leq 117 \\
7 x_{1}+17 x_{2} \leq 207 \\
x_{1}, x_{2} \geq 0
\end{gathered}
$$

Now, using an algorithm for LP problem, the solution of the problem (p2) is

$$
x_{1}=5.19, \quad x_{2}=10.04, \quad Z_{T 2}=40.67
$$

Now, the problem (p1) is given below

$$
\begin{gathered}
(p 1) \max Z_{A 1}=\left(3 y_{1}+4 y_{2}\right) \frac{1}{3} \\
\max Z_{B 1}=\left(5 y_{1}+3 y_{2}\right) \frac{1}{3} \\
\min Z_{C 1}=\left(2 y_{1}+4 y_{2}\right) \frac{1}{3} \\
\max Z_{C 1}=\left(-2 y_{1}-4 y_{2}\right) \frac{1}{3} \\
\left(\text { all max } Z_{T 1}=\left(3 y_{1}+4 y_{2}\right) \frac{1}{3}+\left(5 y_{1}+3 y_{2}\right) \frac{1}{3}+\left(-2 y_{1}-4 y_{2}\right) \frac{1}{3}\right. \\
Z_{T 1}=2 y_{1}+y_{2}
\end{gathered}
$$

s.t

$$
\begin{gathered}
5 y_{1}+4 y_{2} \leq 40 \\
4 y_{1}+14 y_{2} \leq 100 \\
y_{1} \leq 5.19 \\
y_{2} \leq 10.04 \\
y_{1}, y_{2} \geq 0
\end{gathered}
$$

Now, using an algorithm for LP problem, the solution of the problem (p1) is

$$
y_{1}=5.19, \quad y_{2}=3.51, \quad Z_{T 1}=13.89
$$


Now, the problem (p3) is given below

$$
\begin{aligned}
& \text { (p3) } \max Z_{A 3}=\left(12 t_{1}+13 t_{2}\right) \frac{1}{3} \\
& \max Z_{B 3}=\left(14 t_{1}+12 t_{2}\right) \frac{1}{3} \\
& \min Z_{C 3}=\left(9 t_{1}+9 t_{2}\right) \frac{1}{3} \\
& \max Z_{C 3}=\left(-9 t_{1}-9 t_{2}\right) \frac{1}{3} \\
& Z_{T 3}=\left(12 t_{1}+13 t_{2}\right) \frac{1}{3}+\left(14 t_{1}+12 t_{2}\right) \frac{1}{3}+\left(-9 t_{1}-9 t_{2}\right) \frac{1}{3} \\
& Z_{T 3}=5.67 t_{1}+5.33 t_{2} \\
& 15 t_{1}+10 t_{2} \leq 270 \\
& 13 t_{1}+21 t_{2} \leq 420 \\
& t_{1} \geq 5.19 \\
& t_{2} \geq 10.04 \\
& t_{1}, t_{2} \geq 0
\end{aligned}
$$

S.t

Now, using an algorithm for LP problem, the solution of the problem (p3) is

$$
t_{1}=7.95, \quad t_{2}=15.08, \quad Z_{T 3}=125.44
$$

Therefore, the solution for the given MOLP problem with fuzzy objective coefficients, fuzzy technical coefficients and fuzzy variables is

$$
\begin{gathered}
\widetilde{x_{1}}=\left(y_{1}, x_{1}, t_{1}\right)=(5.19,5.19,7.95) \\
\widetilde{x_{2}}=\left(y_{2}, x_{2}, t_{2}\right)=(3.51,10.04,15.08) \\
\widetilde{Z_{T}}=\left(Z_{T 1}, Z_{T 2}, Z_{T 3}\right)=(13.89,40.67,125.44)
\end{gathered}
$$

\section{Conclusion}

Multi-objective linear programming problems with fuzzy interval are considered in this paper. A simple and efficient method can solve such problems. This method can solve problems under consideration by transforming a linear multi - objective to linear single objective and provide an approximation to the optimal solution without using ranking functions and applying classical single linear programming technique. If the multi-objective problem with fuzzy numbers is transforming to the single objective and crisp, the method produced three crisp linear programming problems with the same optimum solutions.

\section{References}

[1] Ahmad, S.U. and Sardar, S.K., 'Fuzzy Variable Linear Programming with Fuzzy Technical Coefficients, pak. J. stat. oper. Res., vol. VIII, No. 4(2012), PP. 839-847.

[2] Bazaraa, M.S., Jarvis, J.J. and Sherali, H.D.,'Linear Programming and Network Flows, John Wiley and Sons, New York(1990).

[3] Bellman, R.E. and Zadeh, L.A., 'Decision Making in a Fuzzy Environment, management sci., vol. 17(1970), pp. 141-164. 
[4] Campos, L. and Verdegay, J.L., 'Linear Programming Problems and Ranking of Fuzzy Numbers, Fuzzy Sets and Systems, vol. 32(1989), pp. 1-11.

[5] Degado, M., Verdegay, J.L. and Vila, M.A., 'A General Method for Fuzzy Linear Programming, fuzzy sets and systems, vol. 29(1989), pp. 21-29.

[6] Kaisa Miettinen, 'On the Methodology of Multiobjective Optimization with Applications, book(1994), pp. 36-41.

[7] Klir, G. and Yuan, B., 'Fuzzy Set and Fuzzy Logic, Prentice-Hall, Inc. Upper Saddle River, New Jersey 07458, USA(1995).

[8] Lai, Y.J. and Hwang, C.L., 'Fuzzy Mathematical Programming Methods and Applications, springer, berlin(1992).

[9] Mahdavi-Amir, N. and Nasseri, S.H., 'Duality Results and A Dual Simplex Method for Linear Programming Problems with Trapezoidal Fuzzy Variables, fuzzy sets and systems, vol. 158(2007), pp. 1961-1978.

[10] Maleki, H.R, Tata, M. and Mashinchi, M., 'Linear Programming with fuzzy variables, fuzzy sets and systems, vol. 109(2000), pp. 21-33.

[11] Nasseri, S.H. and Ardil, E., 'Simplex Method for Fuzzy Variable Linear Programming Problems, world academy of science, engineering and technology, vol. 8(2005), pp. 198-202.

[12] Nasseri, S.H., 'A New Method for Solving Fuzzy Linear Programming by Using Linear Programming, Applied mathematical sciences, vol. 2(2008), pp. 37-46.

[13] Pandian, P. and Jayalakshmi, M., 'A New Method for Solving Integer Linear Programming Problems with Fuzzy Variables, Applied mathematical sciences, vol. 4, No. 20(2010), pp. 997-1004.

[14] Tanaka, H., Okuda, T. and Sai, K.A., 'On Fuzzy Mathematical Programming, the journal of cybernetice, Vol. 3(1974), pp. 37-46.

[15] Verdegay, J.L., 'A Dual Approach to Solve the Fuzzy Linear Programming Problem, fuzzy sets and systems, vol. 14(1984), pp. 131-141. 